\title{
LOW-ENERGY PHYSICS OF HIGH-TEMPERATURE SUPERCONDUCTORS
}

\author{
V.J. EMERY and S. A. KIVELSON ${ }^{2}$ \\ 'Dept. of Phywics Rroolchaven National Laborntory, Upton, NY 11873 \\ ${ }^{2}$ Dept. of Physios UCLA, Los Angeles, Ct, 90024
}

\begin{abstract}
A.BSTRACT. It barkued that the low-energy properties of high temperzture auperconductorn are dominated by the interaction between the mobile boles and a particular class of coilective modes, cormenponding to local largo-amplitude low-enengy tuctuations in the hole density. The lakter are a consequence of the competition between the effects of long-range Coulomb interactions and the cendency of a low concentration of holes in an antiferromngnet to phawe weparnte. The low-energy behavior of the system is govemed by the sarve fixed point se the two-ctinnnel Kondo problem. which inccounts for the "universality" of the proparties of the cuprute muperopaductan. Predictions of the optical propertien and the spin dyatamico are compared with experiment. The pairing rewonanoe of the twochannel Koodo problem gives a mechunim of high temperature waperconductivity with an uncoventional symmetry of the order parameter.
\end{abstract}

\section{INTRODUCTION}

High temperature superconductivity' is robust phenomenon, occurring in a large number of materials containing $\mathrm{CuO}_{2}$ planea. However, despite analytical arguments suggestiug that there is an attractive intersction between charge carriers, aumericul experiments ${ }^{2}$ on a variety of models have so far failed to produce convincing evidence of a significantly entanced pairing susceptibility, .... although the models are believed to include the essential physical interactions. ${ }^{3}$ It is difficult to escape the feeling that something is missing.

We shajl argue that the kug-range Coulomb interaction is the essential piece of physics that has been ignored. In order to fully understand the basic physics of high temperature superconductors it is necessery to take account of the fact that they are doped insulators and retain in some way the memory of the undoped antiferromagnetic stale. We contend liat the competition between the long-range Coulomb interaction and the tendency of holes in an antiferromagnet to phage separake plays a central role in determining how such a system behaves.

We have argued previously, ${ }^{4,5}$ on the basis of analytical and numerical studies of the two dimensional $t-J$ model that a low concentration of holes in an antiferromagnet is ungtable to phase separation into a bole-rich and a bole-deficient phase. The $t-J$ model describes neutral holes (no long-range Coulomb interaction) with hopping amplitude $t$, and local moments with exchange in texaction $J$. It is easy to see that two boles in an antiferromagnet attract each other. At the shortest distances, this attraction arises from the fact that two boles on uearest-neighbor sites break one less antiferromagnetic bond than two far-separaled holes. At larger distances, it is a consequence of the exchange of magnon pairs. In essence, phase separation is the bost way to minimize the zero-point kinetic energy of the electrons and to reconcile the mobility of doped boles with the maintenance of local antiferromagnetic order (which optimizes the zero-point energy for the half-filled band). This behavior is not restricted to the $t-J$ model. Studies of other models in various limits bave ostablished that phase separation is a common behavior of dilute holes in an antiferromagnet. We believe, but have not yet proven, that it is generic, essentially model-independent, behaviox. However, if it were showa that a given model of holes in an antiferromagnet did not exhibit plase separation for a physically reasonable choict of parameters, we would turn to the experimental evidence (discussed below) which implies that there is a strong' local tendency toward phase separation in the high temperature superconductors, and conclude that that there is an important piece of physics missing from that model.

In the presence of the longunge Coulomb repulsion the holes cannot macroscopically phase

1 
separate un less the counterions are mobile. However, so long as the Coulomb interactions are not too stroug, i.e. if the background diecectric constant is large enough, the local tendency toward phase separation will still have important consequences. It is easy to demonstrate that a model with a short-range tendency for phase separation together with long-range Coulomb interactions is highly frustrated. In a cosrse-grained sense, we can represent the hole-rich, bole-poor, and aversge-density phases as different orientations of a local "block spin". The local tendency toward phase separstion is modelled as a stort-range finromagnetic interaction between spins while, the long-range Coulomb interaction corresponds to a long-range antiferromagnetic interaction. The latter is known to be highly frustrating; at the classical level it produces Devil's staircases ${ }^{6}$ and a large degree of metastability. At the quantum level, the frustration is reflected in the existence of spatially-localized, large-amplitude density fluctuations, or collective modes, in which local regions of the bole-rich and hole-poor phases nucleate and disappear. ${ }^{7}$ The large amplitude of the distortion implies that the collective modes are very "heavy" in the sense that they have little dispersion; even a modest disorder will localize them. Once again there is a large vegree of metastability and, associated with it, extremely slow "glass-like" dynamics, ${ }^{7}$ which is manifested via the spin fluctuations as spin freezing.

In looking for these effects in the cuprate superconductors, it is necessary to consider two quite different situations. If the charge donors are mobile on laboratory time scales, they can be dragged along by the holes and preserve charge neutrality so that the holes can actually phase separate. This ocrurs in two special cases: In photo-doped materials ${ }^{8}$ and in oxygen-doped $\mathrm{La}_{2} \mathrm{CuO}_{4-6}$ where the exygen ions remain fairly mobile to low temperatures. ${ }^{9}$ Of course, it is conceivable that the observed phase separation is driven by extraneous factors such as the oxygen chemistry. However we feel that the fact that phase separation occurs in both of the two known cases where the dopants are mobile provides strong experimental support for an electronically driven tendency for phase separation whenever the long-range Coulomb interactions do not prohibit it.

On the other band if the dopant atoms are absolutely frozen, then clearly phase separation can occur only as a short-distance, fluctuation effect, as we have already argued. There are strong indications of such a local fluctuating phase separation in all the superconducting cuprates. Specifically, the most striking evidence is:

a) Photoemission studies ${ }^{10}$ of both $\mathrm{Lia}_{2-x} \mathrm{Sr}_{x} \mathrm{CuO}_{4-\delta}$ and $\mathrm{Nd}_{2-x} \mathrm{Ce}_{x} \mathrm{CuO}_{4}$ show that, as the hole concentration varies, the chemical potential remains within the insulating gap, while the density of states in the gap grows in proportion to the hole concentration. This is precisely the expected behavior for an inhomogeneous system in which insulating and metallic regions coexist in chemical equilibrium: The density of states in the gap arises from the metallic region, whereas the gap features are associated with the insulating regions. Of course photoemission is bayically a high-energy, short-wavelength probe, so it is sufficient to invoke dynamical phase separation in order to account for these experiments.

b) In most of the high temperalure superconductors $(\pi, \pi)$ is not close to a nesting vector of the Fermi surface. ${ }^{11}$ Nevertheless, experiments indicate that the spin susceptibility is peaked in the neighborhood of $(\pi, \pi)$. Nuclear magnetic resonance experiments on $\mathrm{YBa}_{2} \mathrm{Cu}_{3} \mathrm{O}_{7-\delta}$ show that the relaxation rate of the nucleas spins is an order of magnilude larger on $\mathrm{Cu}$ than on oxygen. ${ }^{12,13}$ The current interpretation is that, the $\mathrm{Cu}$ nuclear spin is relaxed by antiferromagnetic fluctuations, which are strongly suppressed at an oxygen nucleus by geometric form factors. ${ }^{13}$ The latter reflect the position of an axygen atom between two $\mathrm{Cu}$ atoms and are given by $\left(1+\cos q_{\mathrm{a}}\right)$ or $\left(1+\cos q_{b}\right)$, both of which vanish when $\left(q_{a}, q_{0}\right)=(\pi, \pi)$, the wave vector for antiferromagnetic order in the insulating phase. ${ }^{13}$ Thus the experiments require that the wave vector at which spin fluctuations peak in the metallic phase is not too different from $(\pi, \pi)$ and, indeed, this is confirmed by neutron scattering experiments ${ }^{34}$ on $\mathrm{YBa}_{2} \mathrm{Cu}_{3} \mathrm{O}_{7 m-\delta}$. From a microscopic point of view, this behavior is not easy to understand, since the wave vector $(\pi, \pi)$ does not appear to be special ${ }^{11}$, and nesting vectors are, if anything, along the $(1,0)$ and $(0,1)$ directions. This surely implies that some features of the antiferromagnetic insulating state are retained even in the beavily-doped metallic regime. Frustrated phase separation naturally accounts for the 
persistence of pronounced structure at $(\pi, \pi)$, whereas theories based on bomogeneous states have greater difticulty.

c) Less direct, but still compelling evidence comes from the optical response, in which spectral features characteristic of the lightly-doped insulator are found to persist in superconducting materials. ${ }^{28-19}$ It has been for.nd by Thomas et al ${ }^{15}$ that the electronic contribution to the optical absorption is quite similar in lightly hole-doped aud electron-doped materials. The salient features are two very broad characteristic absorption peaks in the insulating gap, one with an energy of order $500 \mathrm{meV}$, and the other with an energy of order $100 \mathrm{meV}$. They seem to be present in all the cuprate superconductors, although they may be more or less well-resolved, depending on their widths in the different materials. The transitions are certainly associated with bound charges, since in all cases the optical absorption vanishes as $T$ and $\omega$ tend to 0 .

It is also worth noting that it is quite difficult to produce electronically homogeneous materials. We regard this as a a retlection of the tendency of the system to phase separate locally in response to a fuctuation in the local potential. Whereas a normal metal would screen small local variations in the concentration of dopant ions, the doped antiferromagnet tends to overscreen, thus amplifying the effect of any small inhomogeneity.

In our opinion, these relatively gross features of high temperature superconductors confirm the relevance of phase separation in these materials. But they also suggest an approach to a moredetailed understanding of the phenomenology of the normal state 20,21 and the mecharism of high temperature superconductivity. It is widely recognized that the normaj-state properties of high temperature superconductors imply the existence of a class of low-irequency collective excitations which strongly scatter the craduction electrons. Here we have a rather obvious candidate. The presence of low-frequency collective modes lypically indicates a nearby phase in which these fluctuations condense into a new ordered state. The only known ordered state which overlaps the metallic phases of the cuprate superconductors is the so-called spin-glass phase, in which there are frozen local magnetic noments but no long-range magnetic order. ${ }^{22,23}$ We suggest that, this phase should be regarded as a "cluster spin-glass", in which there is aubstantial local charge inhomogeneity, and the spins in the hole-deficient regions are locally Neel ordered, but with a random direction of the staggered magnetization. Thus the slow density fluctuations in the metal are related to the existence of the nearby cluster spin-glass phase.

We now consider in greater delail the consequences of this picture, and introduce an effective model from which low-energy properties may be calculated.

\section{IMPLICATIONS OF FRUSTRATED PHASE SEPARATION}

The mobile holes create, aunihilate and scalter from the low-energy collective modes. These processes, together with the distribution of energies of the collective modes determine the lowenergy properties of the system. At present, it is not possible to give 3 fully deductive theory of all of these phenomena and their consequences, starting from e.g. an extended Hubbard model with long-range Coulomb interactions. Therefore we proceed in stages, first constructing a lower-level model from which the law-energy physics may be calculated. Such a theory will be describen in detail in a future publication: ${ }^{7}$ for the present, we describe the essential ideas.

\section{A. CHARGE DYNAMICS}

Our approach is to calculace directly the consequences of the interactions between the mobile boles and the collective modes, which domisa! be low-energy behavior of the system and determine the temperalure-dependence of physical quantities. Because the collective modes correspond to local phase separation, they have significant internal structure; in particular, where the hole densi'y is low, we expect behavior characteristic of the antiferromagnetic insulating state. For the internal degrees of freedom, we shall use the single-mode approximation, in which we ignore all internal excited states save one. This approximation is valis both for small clusters 24 and in the thermodyuamic limit. ${ }^{25}$ The single internal mode is a spin.1 excitation with momentum 
$(\pi, \pi)$ and energy $\omega_{g}$. An NMR experiment, of a neutron scatlering experiment which measures the q-integrated intensity of the $(\pi, \pi)$ peak, does not probe the spatial structure of the collective modes. Consequently, for me zy purposes, we may regard them as point-like objects and introduce operators $b_{0}^{\dagger}(\vec{r})$ and $b_{1, \sigma}^{\dagger}(\vec{r})$, which create a collective mode at position $\bar{r}$ in the spin $\rightarrow$ groundstate or in the spin-1, $S_{z}=\sigma=(-1,0,+1)$ excited state respectively. These operators are well-defined in the adiabatic limit, where the fluctuations are either frozen (due to the disorder) or slow compared to the equilibration times of the microscopic degrees of freedom. Since phase separation is driven by the tendency of the antiferromagnetic ground-state to exclude holes, it followg that the existeace of large-amplitude density fluctuations requires that the system be reasonably close to the adiabatic limit. By the same token, the maximum energy scale (ultraviolet cutoff) of the Aluctuations must be of the order of the exchange integral, J. In general, we expect this approximation to be particularly good in low- or moderately-doped materials whereas, in sufficiently heavily-doped materials, corrections to the adiabatic approximation may be more significant. (A more complete discussion will be presented in a forthcoming publication ${ }^{7}$ ).

Ln order to calculate correlation functions, we note that the collective modes are local in space and that there cannot be two at the same point. Moseover, the fact the collective modes correspond to a local charge inhomogeneity implies that they have a dipolar character and that there is a local flow of current as they are created. Using these properties, we have shown ${ }^{7}$ that the scattering of the mobile holes from the collective modes is equivalent to a two-channel Kondo problem. It has already been noted by Cox ${ }^{26}$ that the behavior of the latter has much in common with the phenomenology of the sormal state of high-temperature superconductors. ${ }^{20,21,27}$ When the energy to create a collective mode may be neglected, the imaginary part of the susceptibility for $b_{0}^{\dagger}(\vec{r})$ bas the form ${ }^{28,29}$ :

$$
x^{\prime \prime}(\omega, T)=\frac{1}{2} \tanh (\omega / k T) \frac{r}{\omega^{2}+\Gamma^{2}}
$$

where $\Gamma$ is the Kondo energy scale. We have found that this structure is impressed on many of the observed properties of bigh-temperature superconductors, in particular the optical conductivity 15 and spin fluctuations.

The contribution $\sigma_{L}(\omega)$ to the optical conductivity from the local collective modes may be expressed in terms of this quantity. It is found that: ${ }^{7}$

$$
\sigma_{L}(\omega)=\text { const.w. } x^{n}(\omega)
$$

This gives a peak at $\Gamma$ which should be of the order of the antilerromagnetic exchange integral $\mathrm{J}$. It has been emphasized by Thomas ${ }^{15}$ that there is a mid-infrared peak at this energy in the cuprates at all levels cif doping.

There is considerable agrement on the nature of the optical absorption spectrum in superconducting materials from a few hundreths of an $\mathrm{eV}$ to a few $\mathrm{eV}$, although the interpretation of this data is still controversial. There are two schools of thought: the one-component, or Drude school, ${ }^{16}$ infers a single species of (mobile) charge carrier for which the scallering rate and effective mass depend strongly oni frequency. On the other hand, the two-component or Drude-Lorentz school ${ }^{15,17-19}$ propo: ss that there are two types of charge carrier: mobile charges which dominate the absorption at low frequency and which, at low temperatures, form the su. perconducting condensate; and bound charges which dominate the rnid-infrared absorption but depend weakly on temperature.

In terms of fuctuating phase separation, it is natural to expect the spontaneous generation of a two component absorption spectrum, one associaled with the metallic regions, the other with the insulating (hole-poor) regions. Moreover, it is cleat that the separation into two components should begin to break down when the Drude respouse of the metallic regions extends out to frequeacies characteristic of the density nuctuations. The bound contribution is given in Eq. 
(2). We have also found that, as a consequence of the fluctuating trapping of charge in the local collective modes, the major contribution to the optical conductivity at low frequency has the form:

$$
\sigma_{c}(\omega)=\text { const.Re } \frac{\chi(\omega)}{i \omega}
$$

where the imaginary part of $\chi(\omega)$ is given in Eq. (1). This expression is proportic nal to $\omega^{-1}$ when $\omega$ is larger than $T$, and to $T^{-1}$ when $T$ is lasger than $\omega$, as observed in optimallydoped materials ${ }^{16}$. Note that the frequency-and temperature dependence is attributed to the $\tanh (\omega / 2 k T)$ prefactor in Eq. (1), and not to the scattering rate $\Gamma$.

\section{B. SPIN DYNAMICS}

For a material such as $\mathrm{YBa}_{2} \mathrm{Cu}_{3} \mathrm{O}_{7-\alpha}$ the dyuamical structure factor $S(\omega, T)$ of the operator $b_{1, \pi}^{\dagger}(\vec{r}) b_{0}(\vec{r})$ determines the relaxation rate $T_{1}$ of Cu spins neasured by an NMR experiment and the q-integrated intensity of the $(\pi, \pi)$ peak in neutron scattering. The point is that $(\pi, \pi)$ is not a special nesting vector of the Fermi surface ${ }^{21}$ in $\mathrm{YBa}_{2} \mathrm{Cu}_{3} \mathrm{O}_{7-\delta}$ and thus the amplitude for spin excitations at this wave vector is significant only within the hole-poor part of a collective mode. We have shown ${ }^{7}$ that, when $\omega$ is small compared to $\Gamma, S\left(\omega, T^{\prime}\right)$ is given by

$$
S(\omega, T)=A\left(\frac{\omega_{g}}{k T}\right)\left\{\exp \left(\frac{\omega_{g}}{k T}\right) F\left(\omega-\omega_{g}, T\right)+F\left(\omega+\omega_{g}, T\right)\right]
$$

where

$$
F(\omega, T)=\frac{\pi}{2} \delta(\omega)+\left[\Gamma\left(1+\exp \left(-\frac{\omega}{k T}\right)\right]\right]^{-1}
$$

and

$$
A(x)=\operatorname{const} .[3+e x p(x)]^{-1}
$$

If the excitation energy of the collective modes had been included, it would have broadened the $\delta$-function in Eq. (3).

The relaxation rate of a Cu nucleus ${ }^{63} T_{\downarrow}^{-1}$ is proportional to $S(\omega=0, T)$, where $S(\omega, T)$ is given by Eqs. (4)-(6). It follows that:

$$
{ }^{63} T_{1}=B\left[4+\exp \left(\frac{\omega_{g}}{k T}\right)+3 \exp \left(-\frac{\omega_{g}}{k T}\right)\right]
$$

where $\mathrm{B}$ is a constant. This exprassion provides a quite good description of the temperaturedependence of ${ }^{63} \mathrm{~T}_{1}$ in $Y \mathrm{Ba}_{2} \mathrm{Cu}_{3} \mathrm{O}_{3.63}$ (ref. 12), wilh $\omega_{9}=240 \mathrm{~K}$, and in $Y B a_{2} \mathrm{Cu}_{4} \mathrm{O}_{8}$ (ref. 30), with $\omega_{g}=285 K$. The calculation of the Knight shifl and $T_{1}$ for other nuclei will be presented in a future publication. ${ }^{7}$

Recent neutron-acattering experiments ${ }^{31}$ on $\mathrm{YBa}_{2} \mathrm{Cu}_{3} \mathrm{O}_{7-\delta}$ for $\delta=04$ and $T_{c}=53 \mathrm{~K}$, were fitted to a phesomenological expression for $S(\omega, T)$, similar to that quoted in Eqs. (4) and (5), except that the $\delta(\omega)$ term in $\mathrm{Eq}$. (5) was omitted. It was found that $\omega_{s}=9 \mathrm{meV}$, which is about half as !arge as as the value required to fit the NMIR experiments. As pointed out by Tranquada et al, ${ }^{31}$ this may not be a serious discrepancs, since $\omega_{g}$ varies rapidly with small changes in oxygen content. Moreover, the value of $\omega_{g}$ was determined above $T_{c}$ by $N M R$ but below $T_{c}$ by neutron scattering experiments which, at least initially, were designed to find the superconducting gap. Clearly it is desirable to fit the the results of neutron scattering experiments carried out above $T_{c}$ to the expressions given here. 
Clearly it is desirable to fit the the results of neutron scattering experiments carried out above $T_{c}$ to the expressions given bere.

$\mathrm{I}_{1} \mathrm{a}_{2-x} \mathrm{Sr}_{x} \mathrm{CuO}_{4-\delta}$ is different from $\mathrm{YBa}_{2} \mathrm{Cu}_{3} \mathrm{O}_{7-\delta}$ because there are incommensurate peaks near to $(\pi, \pi)$ which are related to a Fermi surface instability. ${ }^{32}$ Consequently, NMR and neutron scattering experiments on this material do not give direct information about the "doped-insulator" eifects that we have explored here and it is inappropriate to invoke a spin gap. In our picture, any specific frequency- and temperature dependence is obtained because the dynamics of the collective modes are impressed on the motion of the mobile holes. ${ }^{7}$ Experimentally, ${ }^{33}$ the frequencydependence of the q-integrated susceptibility was found to be cousistent with $\arctan (\omega / 2 k T)$. However, within experimental exror, an equally good fit can be obtained using $\tanh (\omega / 2 k T)$, as suggested by $E_{q}$. (1).

The spatial structure of a collective mode may be probed by a q-resolved neutron scattering experiment. The major effect is a cutoff in the q-width of the $(\pi, \pi)$ peak when the correlation length reaches the size of the cluster. The latter may be quite large for lightly-doped materials but is a few lattice spacings for superconducting materials, close to optimum doping.

\section{SUPERCONDUCTIVITY}

An additional feature of the fixed point of the two-channel Kondo problem is an enhancement of the local superconducting susceptibility as a result of a pairing resonance involving the local collective mode and the metallic electrons. Superconductivity appears when coherence develops between nearby regions of the solid. Elsewhere, we and others ${ }^{29,34}$ have examined the nature and symmetry of this resonance. While it is clear that the resulting superconducting order parameter is spin singlet, we have not yet fully explored the remaining symmetry, although it appears ${ }^{29}$ that it may be odd in frequency. ${ }^{36}$

\section{CONCLUSION}

In the preceding sections we have introduced a new way of thinking about the physics of doped insulators and, in particular high temperature superconductors. There are several features of our approach that we feel are worth emphasizing here. First of all, it is clear, that what we have aketched here is not a fully microscopic theory; it starts from an intermediate scale model based on specific localized collective modes. In that sense it is logically independent of the central discussion of the present paper. The connection with frustrated phase separation is that this concept provides an appealing rationale for such a model.

Secondly, despite the formal similarity, the present theory should be distinguished from the onedimensional electron gas where the low-energy behavior is determined by the fixed line of the Tomonaga-Lurtinger model ${ }^{36}$ rather than a fixed paint. The difference is important because the behavior along a fixed line is non-universal, and typically depends on coupling constants, the carrier density, and the details of the short-distance cutoff. It is an essential fealure of our theory that a fixed point determines the behavior of the system in the temperature range between $\Gamma$ and $T_{e}$ (where new coherent phenomena come inco play). This behavior accounts for the robustness and universality of many of the normal-state fealures of the high temperature superconductors.

Thirdly, we point out that the present theory is consisteat with the unusual normal-state properties of high temperalure superconductors. First of all, as was first noted by Cox ${ }^{26}$, the behavior of the two-channel Kondo problem has important similarities to the phenomenology emphasized in the marginal Fermi liquid theory. ${ }^{21,37}$ As a minimum, our theory of the specific low-energy localized collective modes outlined above can be viewed as a higher level model, one step closer to the microscopic, which rationalizes the rnore successful aspects of that phenomenology. Important features of these collective modes are that they are dipolar and that they generate their own (two-channel Kondo) dynamics through their interaction with the mobile holes. Also they have an internal structure, which is especially important in understanding the magnetic properties of the system. Their energy scale is found to be of order J, as required to explain the experiments. 
On the other hand, the collective modes previously invoked in the marginal Fermi liquid theory ${ }^{21}$ were thought to be featureless charge-density excitatious, and their dynamical properties were assumed at the outset. We also note that, although the behavior of the two-channel Kondo problem is marginal in some respects, it is truly non-Fermi liquid in character. ${ }^{34}$ Is particular, the electron self energy is proportional to $(\omega / \Gamma)^{1 / 2}$ at $T=0$.

Finally, it is clear that the present theory satisfies, for the most part, the experimental constraints on any theory of the normal state of high temperature superconductors, outlined recently by Anderson. ${ }^{27}$ In particular, the essential dynamical structure is determined by a fixed point. However, we find enhanced pairing within a single $\mathrm{CuO}_{2}$ plane and do not need to invoke a fundamental role for the tunnelling of electrons between planes.

Brief accounta of some of our ideas have previously been given in conference reports. ${ }^{33}$ More detailed and extended accounts will be presented in papers currently under preparation. ${ }^{7}$

\section{ACKNOWLEDGMENTS}

We benefitted greatly from discussions with more of our colleagues then we can possibly list. However, we must particularly acknowledge useful discussions with G. Aeppli, A. Auerbach, R. Birgeneau, J. Budnick, D.L. Cox, A. Heeger, H.Q. Lin, G. Shirane, J. Tranquada, and A.Tsvelik. Innumerable discussions with Sudip Chakravarty have been central to the development of these ideas, and to our understanding of this problem. SK acknowledges the hospitality of Brookhaven National Laboratory and IBM Almaden Research Center. Part of this work was carried out while VJE was Kramers Professor at The University of Utrecht, The Netherlands, and he thanks Professor J.E. van Himbergen for his hospitality. SK was supported in part by NSF grant \#DMR $-90-11803$. This work also was supported by the Division of Mater' als Sciences, U.S. Department of Energy, under contract DE-AC02-76CH00016.

\section{REFERENCES:}

1. J. G. Bednorz and K. A. Mueller, Z. Phys. B64 (1986) 89.

2. For a review, see D. J. Scalapino in High Temperature Superconductivity, eds. K. S. Bedell, D. Coffey, D.E. Meltzer, D. Pines, and J. R. Schrieffer (Addison-Wesley, Redwood City, 1990) p. 314.

3. P. W. Anderson, Science, 235 (1987) 1196; V. J. Emery, Phys. Rev. Lett. 58 (1987) 2794; C. M. Varma, S. Schmitt-Rink, and E. Abrahams in Novel Superconductivity, eds. S. A. Wolf and V. Z. Kresin (Plenum, New York 1987).

4. V. J. Emery, S. A. Kivelson, and H-Q. Lin, Phys. Rev. Lett. 64 (1990) 475.

5. S. A. Kivelson, V. J. Emery, and H-Q. Lin, Phys. Rev. B 42 (1990) 6523.

6. P. Bak and R. Bruinsma, Phys. Rev. Lett. 49 (1982) 249.

7. V. J. Emery and S. A. Kivelson, to be published.

8. Y. H. Kim et. al., Phys. Rev. B38 (1988) 6478; II. J. Ye et. al., Phys. Rev. B43 (1991) 10574; J. M. Leng et. al., Phys. Rev. B43 (1991) 10582; G. Yu et. al., Phys. Rev. B45 (1992) 4964; D. Mihailovic et al, Phys.Rev. B44 (1991) 237.

9. P. C. Hammel et al, Phys. Rev. B42 (1990) 6781: J.D.Jorgensen et al, Phys.Rev. B38 (1988) 11337.

10. J. W. Allen et al, Phys. Rev. Lett. 64 (1990) 595.

11. W. Picketh, Rev. Mod. Phys. 61 (1989) 433.

12. M. Takigawa et al, Phys. Rev. B43 (1991) 247.

13. For a review, see A. J. Millis in High Temperature Superconductivity, eds. K. S. Bedell, D. Coffey, D.E. Meltzer, D. Pines, and J. R. Schrieffer (.1ddison-Wesley, Redwood City, 1990) p. 198.

14. G. Shirane et al, Phys. Rev. B41 (1990) 6547; J. M. Tranquada et al, Phys. Rev. Lett. 64 (1990) 800; J. Rossat-Mignot et al, Physica B163 (1990) 4.

15. G. Thomas in High Tempcrature Superconductivity, eds. D. P. Tunstall and W. Barford 
(Adam Hilger, Bristol, England, 1991).

16. Z. Schlesinger et al, Phys. Rev. Lett. 65 (1990) 801; L. D. Rotter et al, Phys. Rev. Lett. 67 (1991) 2741 .

17. D. B. Tanner and T. Timusk, University of Florida preprint (1992).

18. Y. Watanabe, Z. Z. Wang, S. A. Lyon, N. P. Ong, D. C. Tsui, J. M. Tarascon, and E. Wang, Princeton University preprint, (1992).

19. C. M. Foster, K. E. Voss, T. W. Hagler, D. Mihailovic, A. J. Ileeger, M. M. Eddy, W. L. Olson, and E. J. Smith, UCSB preprint, (1992).

20. P. W. Anderson, in Frontiers and Borderlines in Many Particle Physics, eds. J. R. Schrieffer and R. Broglia (North Holland, Ansterdam, 1988).

21. C. M. Varma, P. B. Littlewood, S. Schmitt-Rink, E. Abrahams, and A. E. Ruckenstein, Phys. Rev. Lett. 63 (1989) 1996.

22. A. Weidinger et al, Hyp. Int. 63 (1990) 147; B. J. Sternlieb el al., Phys.Rev. B41 (1990) 8866; E. Torikai et al, Hyp. Int., 63 (1990) 271.

23. J. A. Hodges et al, Physica C184 (1991) 259,270; P. Imbert et al, J. de Phys. in press.

24. See, for example F. Figueiesido et al, Phys. Rev. B41 (1989) 4619.

25. S.Chaknavarty, B.I.Halperin, and D.Nelson, Phys. Rev. B39 (1988) 2344.

26. D. L. Cox, unpublished.

27. P. W. Anderson, Science, 256 (1992) 1526.

28. A. M. Tovelick, J. Phys. Condens. Matt. 2 (1990) 2833

29. V. J. Emery and S. Kivelson, preprint.

30. H. Zimmerman et al, Physica C159 (1989) 681.

31. J. M. Tranquada et al, Phys. Rev. B46 (1992): B. J. Sterulieb, M. Sato, S. Shamoto, G. Shirane and J. M. Tranquada, unpublished.

32. P. Littlewood, J. Zaanen, G. Aeppli and H. Monien, unpublished; Y. Zha, Q. Si, and K. Levin, unpublished.

33. T. E. Mason et al, Phys. Rev. Lett. 68 (1992) 1414; T. R. Thurston et al, unpublished.

34. A. W. W. Ludwig and I. Aflleck, Phys. Rev. Lett. 67 (1991) 3160.

35. V. L. Berezinskii, Pisma Zh. Eksp. Teor. Fiz. 20 (1974) 628 [JETP Letl. 20 (1974) 287);

A. Balatsky and E. Abrahams, Phys. Rev. B45 (1992) 13125; E. Abrahams, A. Balatsky, and J. R. Schrieffer, un published.

36. V. J. Emery in Highly Conducting One-Dimensional Solids, eds J. T. De Vreese, R. P. Evrard, and V. E. Van Doren (Plenum, New York, 1979): J. Solyom, Adv. Phys. 28 (1979) 201.

37. A. E. Ruckenstein and C. M. Varma, Physica C185-189 (1991) 134.

38. V. J. Emery, Hyp. Int. 63 (1990) 13; Physica B169 (1991) 17.

\section{DISCLAIMER}

This report was prepared as an account of work sponsored by an agency of the United States Government. Neither the United States Government nor any agency thereof, nor any of their employees, makes any warranty, express or implied, or assumes any legal liability or responsibility for the accuracy, completeness, or usefulness of any information, apparatus, product, or process disclosed, or represents that its use would not infringe privately owned rights. Reference herein 10 any specific commercial product, process, or service by trade name, trademark, manufacturer, or otherwise does not necessarily constitute or imply its endorsement, recommendation, or favoring by the United States Government or any agency thereof. The views and opinions of authors expressed herein do not necessarily state or reflect those of the United States Government or any agency thereof. 

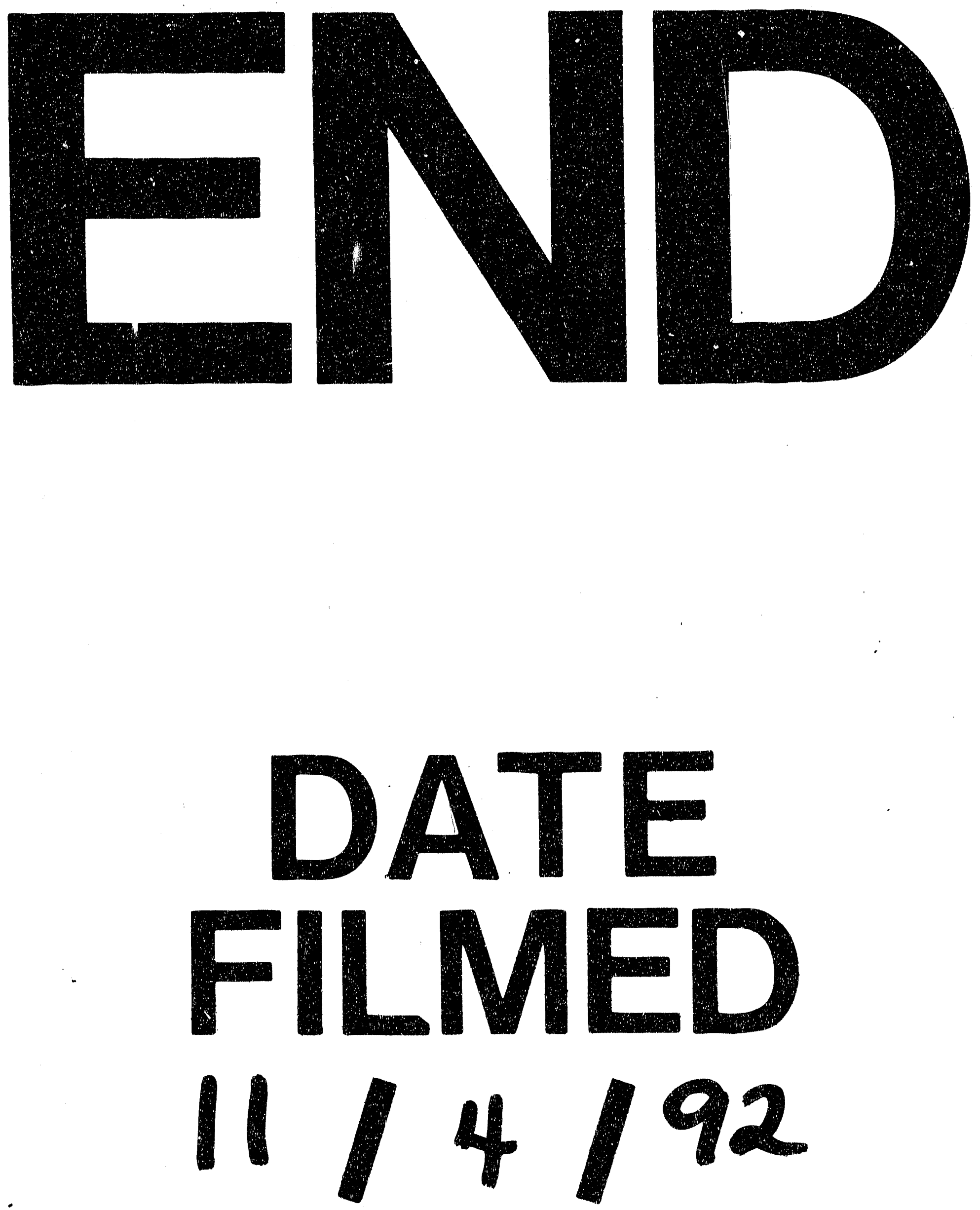

1 
